\title{
Modeling and Analysis of a Manufacturing Plant Using Discrete Event Simulation
}

\author{
Radha Krishna R., Siva Krishna S, Vijay Bhaskar A, Sriram G, Vamsi P*, \\ TVSRK Prasad** \\ *UG Students, Department of Mechanical engineering, VFSTR, Vadlamudi, Guntur Dt, India \\ **Associate Professor, Department of Mechanical Engineering, VFSTR, Vadlamudi, Guntur Dt, India
}

\begin{abstract}
Today's manufacturing systems are characterized by large number of complexities such as random arrival patterns of jobs, random processing times, random failure rates, random repair times, random rejection of parts, etc. The analytical models cannot capture all the randomness mentioned above into the models. There is a need to incorporate them into models to have a practical and real life model. Simulation comes handy in this aspect. Discrete Event Simulation (DES) is used to model a manufacturing system to predict its performance. The inputs to this model include arrival rate, batch size, setup time, processing time, machine breakdown rate, machine breakdown frequency, machines and their capacities, buffers, rejection percentage and inspection time. The outputs that are estimated are work in process, flow time, utilization and throughput.
\end{abstract}

Keywords: Analytical Simulation, Discrete Event Simulation, Random numbers, Optimum process

\subsection{What is Simulation?}

\section{INTRODUCTION}

It is evident that there are many problems of real life which cannot be represented mathematically due to the stochastic nature of the problem, the complexity in problem formulation, or the conflicting ideas needed to properly describe the problem under study. Under such circumstances simulation is often used when all else fail. This method is often viewed as a "method of last resort."

Simulation is the representative model for real situations. In the laboratories we often perform a number of experiments on simulated models to predict the behavior of the real system under true environments. The environments in a museum of natural history and in a geological garden are also good examples of simulation. Actually the idea of simulating real system for enjoyment purposes is already known to us. The chess-playing game is a non-probabilistic simulation of a flight between black and white armies. The game of snake and ladders was initially proposed to simulate the moral progress of the players who moved up ladders when they were 'good' and fell down snakes, indicating temptation, when they were bad. Like in many other board games, dice are used as random number generators.

In all these examples, we have tried to represent the reality to observe- what would happen under real operating situations. Thus, such representation to reality, which may be either in physical form or in a mathematical equations form, may be called simulation. A simulation model mainly consists of two basic phases:

Phase 1: Data Generation. Data generation involves the sample observation of variables and can be carried out with the help of any of the fallowing methods :

(i) Using the random tables:

(ii) Restoring to mechanical devices

(iii) Using electronic computers.

Phase 2: Book-Keeping. The book-Keeping phase of simulation model deals with updating the system when new event occur.

\subsection{What is Excentre?}

It is an important part in piston pump, whose job is to convert rotary motion to reciprocating motion of piston. As shown below, its surface is grinded to mirror finish for smooth operation for over years.

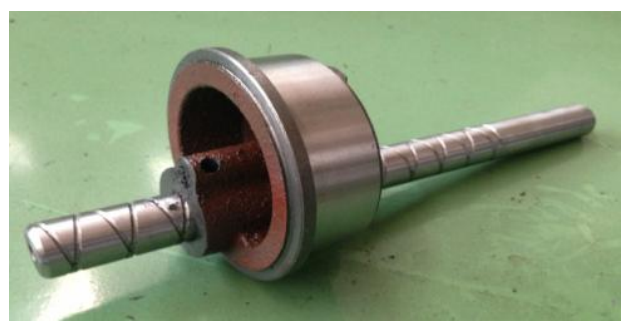

Fig 1. Completely finished Excentre

The manufacturing of Excentre starts in foundry department which includes casting, chipping, red oxide. This later moves to machining department which include turning, drilling, grinding, shaft assembly and inspection. Every stage is 
followed by inspection ensuring only quality products leave the plant. Each stage has a rejection percentage of $1-2 \%$. The monthly production of Excentre was 800-900. Everyday 50-60 components are manufactured on an average. These components move from stage to stage as a batch with size of 50 .

\section{LITERATURE SURVEY}

Simulation is a powerful tool for solving many problems, particularly in manufacturing $[1,2]$. Its use in the modeling and analysis of manufacturing systems are one of its largest application areas, which have become increasingly important in the last couple of decades [3, 4].

Quantitative and qualitative benefits have been attributed to simplifications, and experienced by organizations [2]. Quantitative benefits typically include: reduction in operating costs, throughput time, capital costs, design-to-market time and faster implementation of plant changes. Qualitative benefits typically include: reduction in risk, greater understanding of processes, improvements in communication, better team integration and better development of skills within the organization [2,3].

Simulations allow various issues within manufacturing to be addressed without the drawbacks of experimenting with a real manufacturing system. Typical issues addressed are: the need for and the quantity of equipment and personnel, performance evaluation, and evaluation of operational procedures $[2,5]$.

The adoption of simulations have had limited application within small and medium Enterprises (SME) for many years due to the cost of computing power and lack of computer literacy. As time has passed, improvements in hardware and availability of affordable user friendly tools have enabled computer simulation to be frequently used to address a wide variety of operational problems [6]. The case study presented in this paper is representative of an SME with less than 100 employees.

\section{ANALYSIS OF EXCENTRE}

The below flow chart shows the sequence of operations performed to manufacture an excentre.

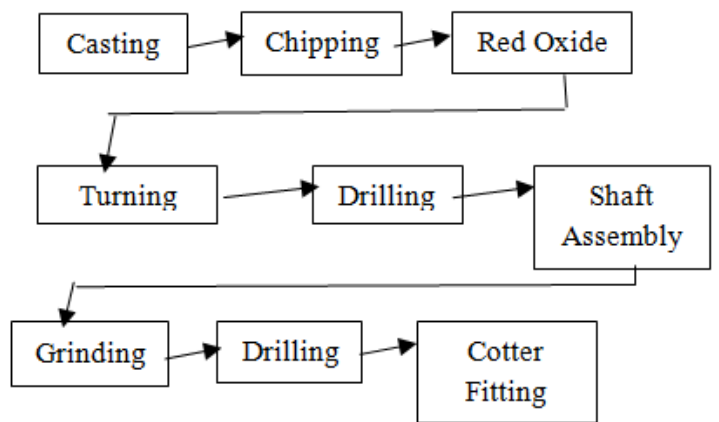

Fig 2. Process flow of Excentre
A sample of data is collected at each stage of manufacture. The sample size (number of observations to be taken) at a particular stage depends on accuracy required.

Types of data: - The following data were collected at various stages of manufacture

- Processing time

- Setup time

- Machine breakdown probability

- Machine repair time

- Fraction of parts accepted/rejected

\section{Casting:-}

Setup time- 20sec, 18, 22, 21, 20, 20, 20, 18, 21, 23, 20, 21, 21, 19, 20 and 22

Processing time- 30sec, 32, 29, 30, 27, 30, 32, 33, $31,32,29,28,33,30$ and 29

\section{Turning:-}

Setup time- 10 sec, 13, 12, 13, 13, 10, 14, 15, 16, 15, $15,14,13,12,11$ and 15

Processing time- 230sec, 231, 233, 236, 237, 239, 243, 245, 246, 249, 230, 233, 237, 243, 249 and 243

Machine repair time- $10800 \mathrm{sec}, 11000,11243$, 10933, 11450, 11640, 12100, 12010, 12145 and 12000

In the same manner, the data for all the processes is collected.

\subsection{Standard Error:}

The standard error gives an idea about the reliability and precision of a sample. The smaller the SE, the greater the uniformity of sampling distribution and hence, greater is the reliability of sample. Conversely, the greater the SE, the greater the difference between observed and expected frequencies. In such a situation, the unreliability of sample is greater. The size of SE depends upon the sample size to a greater extent and it varies inversely with the size of the sample.

$$
\mathrm{SE}=\sqrt{\mathrm{P}} . \mathrm{Q}(1 / \mathrm{n} 1+1 / \mathrm{n} 2)
$$

Where, $\mathrm{P}=(\mathrm{n} 1 \mathrm{p} 1+\mathrm{n} 2 \mathrm{p} 2) /(\mathrm{p} 1+\mathrm{p} 2)$

$$
\begin{aligned}
& \mathrm{Q}=1-\mathrm{p} \\
& \mathrm{N} 1=\text { number of events in sample one }=16 \\
& \mathrm{~N} 2=\text { number of events in sample two }=50
\end{aligned}
$$

For casting,

Mean of setup time from sample data $=20.37=$ $20.37 / 100=0.203=p 1$

Mean of setup time from simulated data $=20.56=$ $20.56 / 100=0.205=\mathrm{p} 2$

$$
\begin{aligned}
\mathrm{P} & =(0.203 * 16+0.205 * 50) /(50+16) \\
& =0.2045 \\
\mathrm{Q} & =1-\mathrm{P} \\
& =1-0.2045=0.795
\end{aligned}
$$

Therefore, $\mathrm{SE}=\sqrt{ }(0.2045 * 0.795) *(1 / 16+1 / 50)$

$$
=0.1158<0.5
$$

Since the error calculated is small, the number of observations is sufficient. 


\subsection{Sample Analysis:}

A sample simulation is performed for casting as shown below:

Table 1: Casting setup time

\begin{tabular}{|l|l|l|l|l|}
\hline Time & Frequency & Percentage & Cumulative \% & Range \\
\hline 18 & 2 & 12 & 12 & $00-11$ \\
19 & 1 & 6 & 18 & $12-17$ \\
20 & 6 & 38 & 56 & $18-55$ \\
21 & 4 & 25 & 81 & $56-80$ \\
22 & 2 & 13 & 94 & $81-93$ \\
23 & 1 & 6 & 100 & $94-99$ \\
\hline
\end{tabular}

Table 2: Casting processing time

\begin{tabular}{|l|l|l|l|l|}
\hline Time & Frequency & Percentage & Cumulative $\%$ & Range \\
\hline 27 & 2 & 13 & 13 & $00-12$ \\
28 & 1 & 6 & 19 & $13-18$ \\
29 & 3 & 19 & 38 & $19-37$ \\
30 & 4 & 25 & 63 & $38-62$ \\
31 & 1 & 6 & 69 & $63-68$ \\
32 & 3 & 19 & 88 & $69-87$ \\
33 & 2 & 12 & 100 & $88-99$ \\
\hline
\end{tabular}

Table 3: Casting Simulation

\begin{tabular}{|c|c|c|c|c|c|c|c|c|}
\hline AT & I AT & RN & ST & RN & PT & MIRT & INS RN & INS \\
\hline $7: 00: 00$ & - & 39 & 20 & 69 & 32 & $7: 00: 52$ & 05 & $\mathrm{Y}$ \\
\hline $7: 00: 52$ & 52 & 73 & 21 & 60 & 30 & $7: 01: 43$ & 89 & $\bar{Y}$ \\
\hline $7: 01: 43$ & 51 & 72 & 21 & 47 & 30 & $7: 02: 34$ & 18 & $\mathrm{Y}$ \\
\hline $7: 02: 34$ & 51 & 75 & 21 & 21 & 29 & $7: 03: 24$ & 08 & $\mathrm{Y}$ \\
\hline $7: 03: 24$ & 50 & 37 & 20 & 06 & 27 & $7: 04: 04$ & 26 & $\bar{Y}$ \\
\hline $7: 04: 04$ & 40 & 02 & 18 & 83 & 32 & $7: 04: 58$ & 47 & $\mathrm{Y}$ \\
\hline $7: 04: 58$ & 54 & 87 & 22 & 49 & 30 & $7: 05: 50$ & 94 & $\mathrm{Y}$ \\
\hline $7: 05: 50$ & 52 & 98 & 23 & 39 & 30 & $7: 06: 43$ & 06 & $\mathrm{Y}$ \\
\hline $7: 06: 43$ & 53 & 10 & 18 & 16 & 28 & $7: 07: 29$ & 72 & $\mathrm{Y}$ \\
\hline $7: 07: 29$ & 46 & 47 & 20 & 13 & 28 & $7: 08: 17$ & 40 & $\mathrm{Y}$ \\
\hline $7: 08: 17$ & 48 & 93 & 22 & 04 & 27 & $7: 09: 06$ & 62 & $\mathrm{Y}$ \\
\hline $7: 09: 06$ & 49 & 21 & 20 & 99 & 33 & $7: 09: 59$ & 47 & $\bar{Y}$ \\
\hline $7: 09: 59$ & 53 & 95 & 23 & 83 & 32 & $7: 10: 54$ & 68 & $\mathrm{Y}$ \\
\hline $7: 10: 54$ & 55 & 97 & 23 & 81 & 32 & $7: 11: 49$ & 60 & $\mathrm{Y}$ \\
\hline $7: 11: 49$ & 55 & 69 & 21 & 74 & 32 & $7: 12: 42$ & 88 & $\bar{Y}$ \\
\hline $7: 12: 42$ & 53 & 41 & 20 & 93 & 33 & $7: 13: 35$ & 17 & $\mathrm{Y}$ \\
\hline $7: 13: 35$ & 53 & 91 & 22 & 74 & 32 & $7: 14: 39$ & 36 & $\mathrm{Y}$ \\
\hline $7: 14: 39$ & 64 & 80 & 21 & 85 & 32 & $7: 15: 32$ & 77 & $\mathrm{Y}$ \\
\hline $7: 15: 32$ & 53 & 76 & 21 & 84 & 32 & $7: 16: 25$ & 43 & $\bar{Y}$ \\
\hline $7: 16: 25$ & 53 & 59 & 21 & 82 & 32 & $7: 17: 18$ & 28 & $\mathrm{Y}$ \\
\hline $7: 17: 18$ & 53 & 63 & 21 & 36 & 29 & $7: 18: 08$ & 31 & $\mathrm{Y}$ \\
\hline $7: 18: 08$ & 50 & 78 & 21 & 96 & 33 & $7: 19: 02$ & 06 & $\bar{Y}$ \\
\hline $7: 19: 02$ & 54 & 87 & 22 & 72 & 32 & $7: 19: 56$ & 58 & $\mathrm{Y}$ \\
\hline $7: 19: 56$ & 54 & 47 & 20 & 05 & 27 & $7: 20: 43$ & 39 & $\mathrm{Y}$ \\
\hline $7: 20: 43$ & 47 & 56 & 20 & 37 & 29 & $7: 21: 32$ & 71 & $\mathrm{Y}$ \\
\hline $7: 21: 32$ & 49 & 22 & 20 & 22 & 29 & $7: 22: 31$ & 22 & $\mathrm{Y}$ \\
\hline $7: 22: 31$ & 59 & 19 & 20 & 32 & 29 & $7: 23: 30$ & 76 & $\mathrm{Y}$ \\
\hline $7: 23: 30$ & 59 & 16 & 19 & 34 & 29 & $7: 24: 08$ & 81 & $\mathrm{Y}$ \\
\hline $7: 24: 08$ & 38 & 78 & 21 & 63 & 31 & $7: 25: 08$ & 8.8 & $\mathrm{Y}$ \\
\hline $7: 25: 08$ & 60 & 03 & 18 & 16 & 28 & $7: 25: 54$ & 94 & $\mathrm{Y}$ \\
\hline $7: 25: 54$ & 46 & 04 & 18 & 99 & 33 & $7: 26: 45$ & 76 & $\mathrm{Y}$ \\
\hline $7: 26: 45$ & 51 & 61 & 21 & 77 & 32 & $7: 27: 38$ & 23 & $\mathrm{Y}$ \\
\hline $7: 27: 38$ & 53 & 23 & 20 & 93 & 33 & $7: 28: 31$ & 47 & $\mathrm{Y}$ \\
\hline $7: 28: 31$ & 53 & 15 & 19 & 52 & 30 & $7: 29: 20$ & 25 & $\mathrm{Y}$ \\
\hline $7: 29: 20$ & 49 & 58 & 21 & 23 & 29 & $7: 30: 10$ & 79 & $\bar{Y}$ \\
\hline $7: 30: 10$ & 50 & 93 & 22 & 63 & 31 & $7: 31: 13$ & 08 & $\mathrm{Y}$ \\
\hline $7: 31: 13$ & 63 & 78 & 21 & 70 & 32 & $7: 32: 19$ & 15 & $\mathrm{Y}$ \\
\hline $7: 32: 19$ & 66 & 61 & 21 & 25 & 29 & $7: 33: 28$ & 71 & $\mathrm{Y}$ \\
\hline $7: 33: 28$ & 69 & 42 & 20 & 65 & 31 & $7: 34: 47$ & 58 & $\bar{Y}$ \\
\hline $7: 34: 47$ & 79 & 77 & 21 & 99 & 33 & $7: 35: 41$ & 56 & $\mathrm{Y}$ \\
\hline $7: 35: 41$ & 54 & 65 & 21 & 64 & 31 & $7: 36: 33$ & 31 & $\mathrm{Y}$ \\
\hline $7: 36: 33$ & 52 & 71 & 21 & 12 & 27 & $7: 37: 21$ & 11 & $\mathrm{Y}$ \\
\hline $7: 37: 21$ & 48 & 20 & 20 & 97 & 33 & $7: 38: 14$ & 94 & $\mathrm{Y}$ \\
\hline $7: 38: 14$ & 53 & 17 & 19 & 78 & 32 & $7: 39: 05$ & 31 & $\mathrm{Y}$ \\
\hline $7: 39: 05$ & 49 & 48 & 20 & 34 & 29 & $7: 39: 54$ & 88 & $\bar{Y}$ \\
\hline $7: 39: 54$ & 51 & 89 & 22 & 29 & 29 & $7: 40: 45$ & 10 & $\mathrm{Y}$ \\
\hline $7: 40: 45$ & 51 & 18 & 19 & 12 & 27 & $7: 41: 31$ & 15 & $\mathrm{Y}$ \\
\hline $7: 41: 31$ & 46 & 83 & 22 & 59 & 30 & $7: 42: 23$ & 54 & $\mathrm{Y}$ \\
\hline $7: 42: 23$ & 52 & 08 & 18 & 48 & 30 & $7: 43: 11$ & 77 & $\mathrm{Y}$ \\
\hline $7: 43: 11$ & 48 & 90 & 22 & 50 & 30 & $7: 44: 03$ & 24 & $Y$ \\
\hline
\end{tabular}

\section{Abbrevations:}

$\begin{array}{ll}\text { AT } & \text { Arrival Time } \\ \text { I AT } & \text { Inter Arrival Time } \\ \text { ST } & \text { Setup Time } \\ \text { PT } & \text { Processing Time }\end{array}$




$\begin{array}{ll}\text { FT } & \text { Flow Time } \\ \text { MRT } & \text { Material Removal Time } \\ \text { RN } & \text { Random Number } \\ \text { BD1 RN } & 1^{\text {st }} \text { machine Breakdown Random Number } \\ \text { BD1 RT RN } & 1^{\text {st }} \text { machine Repair Time Random Number } \\ \text { BD1 RT } & 1^{\text {st }} \text { machine Repair Time } \\ \text { BD2 RN } & 2^{\text {nd }} \text { machine Breakdown Random Number } \\ \text { BD2 RT RN } & 2^{\text {nd }} \text { machine Repair Time Random Number } \\ \text { BD2 RT } & 2^{\text {nd }} \text { machine Repair Time } \\ \text { INS RN } & \text { Inspection Random Number } \\ \text { INS } & \text { Inspection }\end{array}$

\subsection{Model Validation:}

In many decision-situations, we may be interested in knowing whether the parameters of two populations are alike or different. We shall explain now the technique of hypothesis testing for differences between means.

$$
\mathrm{Z}=\left(\mathrm{X}_{1}-\mathrm{X}_{2}\right) / \sqrt{ } \sigma^{2}\left(1 / \mathrm{N}_{1}\right)+\left(1 / \mathrm{N}_{2}\right)
$$

Mean of setup time from sample data, $X_{1}=20.37$

Mean of setup time from simulated data, $X_{2}=20.56$

Sigma $=$ Average of standard deviation of setup time

$$
\begin{aligned}
& =(2.59+2.5) / 2 \\
& =2.5
\end{aligned}
$$

$\mathrm{N}_{1}=$ number of events in sample one $=16$

$\mathrm{N}_{2}=$ number of events in sample two $=50$

$$
\begin{aligned}
\mathrm{Z} & =(20.56-20.37) / \sqrt{2} .5^{2}(1 / 16+1 / 50) \\
& =0.278<1.96
\end{aligned}
$$

Since the value of $\mathrm{Z}$ calculated (i.e., 0.278) is less than table value of $\mathrm{Z}$ (i.e., 1.96), it can be inferred that the model is adequate with $95 \%$ confidence level.

\section{RESULTS AND DISCUSSIONS}

The fallowing system outputs are calculated using simulation.

- Throughput

- Utilization

- Flow time

- Work In Process

The above outputs are an indication of the systems performance measures. The above performance measures are calculated for each stage of manufacture and also for entire system.

For an easy understanding, the fallowing lines will give an idea to judge the performance of the system.

1. Utilization should be high

2. Throughput should be high

3. Flow time should be small

4. Work In Process should be small.

\subsection{Casting Results:}

1) Average number of parts in system

$$
\begin{aligned}
& =(50 * 32)+(49 * 30)+ \\
& =26 \text { parts } / \mathrm{sec}
\end{aligned}
$$

2) Average flow time

$$
=32+62+92+121 \ldots . .+1520 \div 50
$$

$=776 \mathrm{sec} / \mathrm{part}$

3) Total of the inter arrival times $=52+51+51+50+\ldots .$. $=2591 \mathrm{sec}$

4) Mean of the inter arrival times $=2591 / 50$ $=51.8 \mathrm{part} / \mathrm{sec}$

5) Arrival rate $=50 \div 2591$ $=0.01991 \mathrm{part} / \mathrm{sec}$

6) Total service time $=1520 \mathrm{sec}$

7) Average service time $=1520 \div 50$ $=30.4 \mathrm{sec} /$ part

8) Service rate $=1 \div 30.4$ $=0.03289 \mathrm{part} / \mathrm{sec}$

9) Utilization $=$ Arrival rate $\div$ Service rate $=0.5864$

10) Throughput $=50 * 60 * 60 \div 2643$ $=68 \mathrm{part} / \mathrm{hr}$

11) Work in process $=50$ parts Like above process, results will be calculated for chipping, red oxide, turning, etc.,

\subsection{Overall Results:}

1) Average number of parts in system $=65952+27937+\ldots . .+7151 \div$ $=22 \mathrm{part} / \mathrm{sec}$

2) Over all flowtime $=776+1374+621+\ldots \ldots+179$ $=15570 \mathrm{sec} / \mathrm{part}$

3) Total of the inter arrival times $=2591+3018+\ldots=39250 \mathrm{sec}=11 \mathrm{hrs}$

4) Avg arrival rate $=0.0191 \mathrm{parts} / \mathrm{sec}$

5) Service time $=30408 \mathrm{sec}=8.4 \mathrm{hrs}$

6) Service rate $=0.0949 \mathrm{part} / \mathrm{sec}$

7) Utilization $=69.1 \%$

8) Overall Throughput $=69.5 \mathrm{part} / \mathrm{hr}$

9) $\mathrm{Avg} \mathrm{WIP}=43.8$ parts 


\subsection{Results at a glance:}

Table 4: Overall Results

\begin{tabular}{|l|l|l|l|l|l|l|l|l|}
\hline Process & $\begin{array}{l}\text { Parts in } \\
\text { sys }\end{array}$ & $\begin{array}{l}\text { Flow } \\
\text { time }\end{array}$ & I AT & M AT & $\begin{array}{l}\text { Service } \\
\text { time }\end{array}$ & Utilization & Throughput & WIP \\
\hline Casting & 25 & 775 & 2591 & 52 & 1520 & 58 & 68 & 50 \\
Chipping & 24 & 1374 & 3018 & 63 & 2679 & 86 & 57 & 48 \\
Red oxide & 22 & 620 & 2108 & 47 & 1221 & 58 & 76 & 45 \\
Turning & 22 & 5366 & 11340 & 258 & 10505 & 89 & 14 & 44 \\
Drilling & 22 & 2176 & 6496 & 151 & 4235 & 65 & 23 & 43 \\
Shaft-ass & 21 & 271 & 1043 & 24 & 540 & 53 & 145 & 43 \\
Grinding & 22 & 2345 & 5866 & 136 & 4584 & 77 & 25 & 43 \\
Drilling & 21 & 1174 & 3249 & 80 & 2299 & 70 & 45 & 41 \\
Cotter & 22 & 1292 & 2797 & 68 & 2466 & 88 & 52 & 41 \\
Inspection & 20 & 179 & 742 & 19 & 359 & 47 & 190 & 40 \\
\hline
\end{tabular}

UNITS:

Flow time(sec/part) I AT(sec) M AT(sec/part) Service time(sec) Throughput(Parts/hr) WIP(parts)

\subsection{Graphs:}

Based on the above results, the following graphs are drawn to find out the optimum parameters in each stage. The following are NONDOMENATED or PARETO OPTIMAL points i.e., each point has equal importance with the other, but we can't make a conclusion which is optimum among those. If a point is good on one objective, the other pareto optimal point is good on other objective.

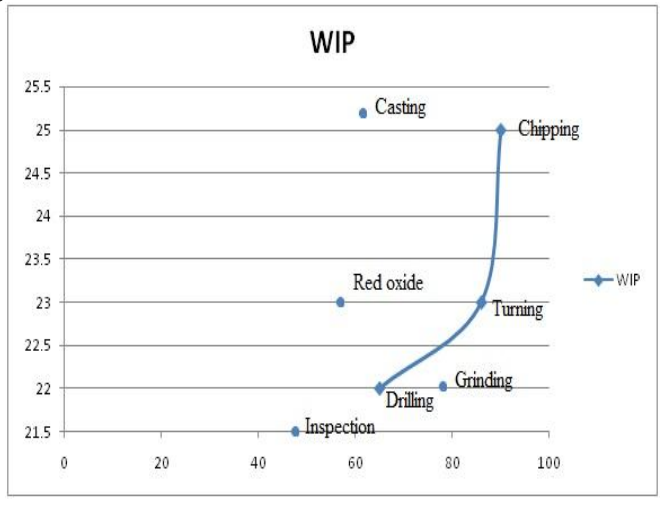

Fig 3: Utilization vs. WIP

Note: Processes chipping, turning and drilling are efficient from the above plot. The remaining processes are not efficient; when the utilization and WIP are taken as the objectives.

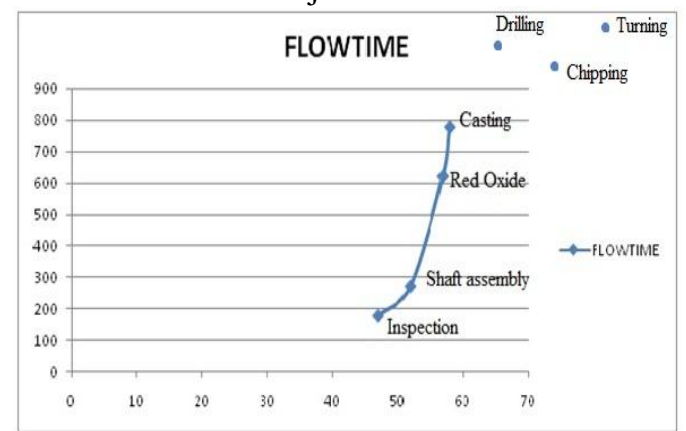

Fig 4: Utilization vs. Flow time
Note: Processes casting, Red oxide, Shaft assembly, Inspection are efficient from the above plot. The remaining processes are not efficient; when the utilization and flow time are taken as the objectives.

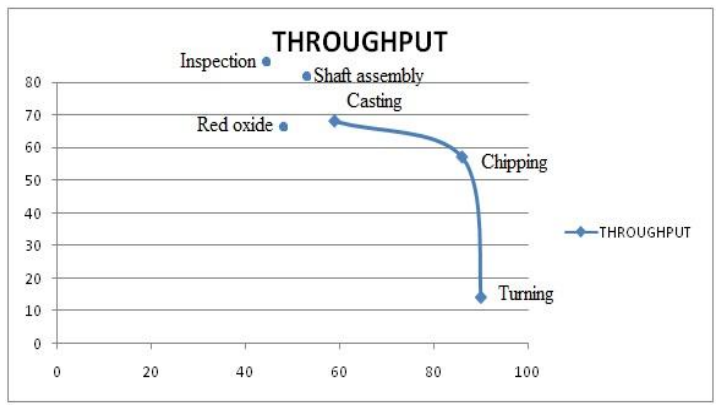

Fig 5: Utilization vs. Throughput

Note: Processes casting, chipping and turning are efficient from the above plot. The remaining processes are not efficient; when utilization and throughput are taken as objectives.

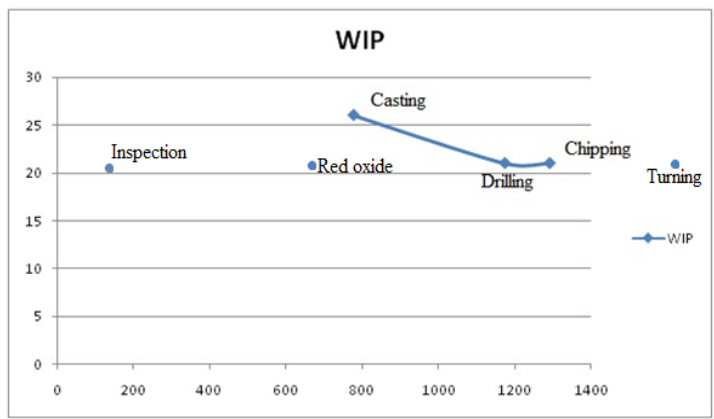

Fig 6: Flow time vs. WIP

Note: Processes Casting, Drilling and chipping are efficient from the above plot. The remaining processes are not efficient; when flow time and WIP are taken as objectives. 


\subsection{Suggestions:}

Setup time is more in turning. To reduce it some fixtures can be designed and implemented. Utilization is less in inspection. To improve utilization, the inspector may be assigned more tasks than present.

\section{CONCLUSION}

Discrete event simulation model was designed for a manufacturing plant. The model was validated by collecting data and conducting hypothesis test. It was found that the model was valid at $95 \%$ confidence level.

Simulation was performed for 50 pieces and various performance measures were determined. Finally, the various processes were compared regarding efficiency. This is known as benchmarking. Various graphs showing performance measures were plotted and pareto optimal front was marked.

\section{SCOPE FOR FUTURE WORK}

In the present work, hand simulation was done for one part called Ex-centre of Reciprocating pump. The results obtained in this work may be compared with analytical model like queueing model. Also the results may be compared with arena software.

Because of lack of time, we have limited our work to hand simulation only. In future, this work can be extended to include all parts of reciprocating pump and also all other pumps such as submersible pump, centrifugal pump etc, made by the company, using arena software. Also in future, it is proposed to determine the cost of each product also by using simulation.

\section{REFERENCES}

[1] Jeffrey W. Herrmann, Edward Lin, Bala Ram, Sanjiv Sarin, Adaptable simulation models for manufacturing, Proceedings of the 10th International Conference on Flexible Automation and Intelligent Manufacturing, Volume 2, pp. 989-995, College Park, Maryland, June 26-28, 2000

[2] B.W. Hollocks, The impact of simulation in manufacturing decision making, Control Eng. Practice, Vol. 3, No. 1, pp. 106112,1995

[3] F. Hosseinpour, and H. Hajihosseini, Importance of Simulation in Manufacturing; World Academy of Science, Engineering and Technology 51 2009

[4] Y.G. Sandanayake, C.F. Oduoza, D.G. Proverbs, A systematic modeling and simulation approach for JIT performance optimisation, Robotics and Computer-
Integrated Manufacturing 24 (2008) 735743

[5] S. Andradottir, K.J. Healy, D.H. Withers, and B.L. Nelson, Simulation of manufacturing systems, Proceedings of the 1997 Winter Simulation Conference

[6] Methodology for rapid identification and collection of input data in the simulation manufacturing systems, Simulation Practice and Theory 7 (2000) 645-656

[7] Simulation modeling and Analysis, Averill M Law ( TATA Mc Graw Hill, 4th ed)

[8] Discrete event system simulation, Jerry Banks(pearson,4th ed) 